\title{
Multimodal magnetic resonance imaging increases the overall diagnostic accuracy in brain tumours: Correlation with histopathology
}

\section{K Abul-Kasim, M Thurnher, S Puchner, P Sundgren}

Faculty of Medicine, Lund University, Division of Neuroradiology, Diagnostic Centre for Imaging and Functional Medicine, Skåne University Hospital, Malmö, Sweden

K Abul-Kasim, MD, PhD

Faculty of Medicine, Lund University, Division of Neuroradiology, Centre for Medical Imaging and Physiology, Skåne University Hospital, Lund, Sweden P Sundgren, $\mathrm{MD}, \mathrm{PhD}$

Department of Radiology, Medical University of Vienna, Austria

M Thurnher, MD, PhD

S Puchner, MD

Corresponding author: K Abul-Kasim (kasim.abul-kasim@med.lu.se)

\begin{abstract}
Background. The aim of this retrospective study was to assess the contribution of multimodal MRI techniques, specifically perfusion-weighted imaging (PWI), and/or MR spectroscopy (MRS), in increasing the diagnostic accuracy of MRI in brain tumours.

Methods. Forty-four patients with suspected brain tumours (27 (61\%) patients male, mean age 58 (standard deviation \pm 17 ) years) were included in this retrospective analysis. Patients were examined with conventional MR sequences, DWI, and with PWI and/or MRS. The concordance between the diagnoses obtained with multimodal MRI and with the conventional MR sequences, and the final diagnosis obtained by biopsy, was estimated. Fisher's exact test and/or chi-square test was performed to estimate the added utility of multimodal MRI. Statistical significance was set at $p<0.05$. Results. With multimodal MRI, the diagnosis in 41 (93\%) patients was the same as that obtained by biopsy, compared with 39\% (17/44) patients when the readers were allowed to give one diagnostic possibility during the evaluation of the conventional MR sequences alone $(p<0.001)$. The concordance between the diagnoses provided by evaluating the multimodal MRIs and the final diagnoses was almost perfect ( $\kappa$ value $0.92,95 \%$ CI 0.82 - 1.0). PWI primarily helped to differentiate lymphomas from other solid tumours, whereas MRS helped to differentiate malignant glioma from metastasis. Both PWI and MRS helped in grading astrocytomas.

Conclusion. Multimodal MRI increases diagnostic accuracy and should, wherever available, be performed in the work-up of brain tumours, although this entails increased examination cost and time.
\end{abstract}

S Afr J Rad 2013;17(1):4-10. DOI:10.7196/SAJR.812

Treatment strategies differ significantly from one type of brain tumour to another. Furthermore, brain biopsy is an invasive procedure and, unlike a biopsy taken from other organs, means a major operative procedure. Every effort should be made to non-invasively establish an accurate preoperative diagnosis to help in treatment planning and determining the prognosis of brain tumours. Even with aggressive treatment strategies, including surgical resection, along with recent advances in radiotherapy and chemotherapy, the prognosis for glioblastoma multiforme (GBM) remains poor, with a median survival of about 14 months after diagnosis. ${ }^{[1]}$ Surgery is the cornerstone of treatment for the majority of low-grade astrocytomas, while postoperative radiotherapy has been shown to lengthen the progression-free period without significantly affecting the overall survival - median survival is 7.2 7.4 years. ${ }^{[2]}$ Solitary metastases that are surgically accessible must be differentiated from malignant glioma. Among the tumours that need to be diagnosed non-invasively are those that are not accessible to biopsy or surgery, and those that can primarily be treated with radiotherapy and/or cytotoxic therapy, such as lymphomas. Providing an accurate, non-invasive diagnosis of such tumours could result in less invasive procedures (such as brain biopsy) and enable rapid initiation of tumour therapy, which would not only benefit patients but also reduce overall costs.

Conventional MR sequences provide mainly anatomical and structural information about the relation of a brain tumour to the surrounding tissue, and may help to differentiate brain tumours from other central nervous system (CNS) pathologies. The introduction of new modalities in MRI, such as diffusion-weighted imaging (DWI), perfusion-weighted imaging (PWI), MR spectroscopy (MRS) and, more recently, diffusion tensor imaging (DTI) and functional MRI (fMRI), has significantly improved the possibilities of better characterisation of brain tumours. ${ }^{[3-7]}$ MRS provides additional information about the metabolic properties of tumours and surrounding brain tissue, whereas PWI provides information about the blood volume (CBV) of the tumours and the peritumoural oedema. 


\section{ORIGINAL ARTICLE}

Table 1. Diagnosis given at the evaluation of conventional MR sequences, multimodal MRI and final diagnosis of the whole study population

\begin{tabular}{|c|c|c|c|c|c|}
\hline No. & Diagnosis CT/MR & $\begin{array}{l}\text { Diagnosis when PWI, } \\
\text { MRS added }\end{array}$ & $\begin{array}{l}\text { Added } \\
\text { utility }\end{array}$ & Modalities & Final diagnosis \\
\hline 4 & Metastasis/GBM ${ }^{\star}$ & GBM & Yes & PWI, MRS & GBM \\
\hline 5 & Astrocytoma, low-grade ${ }^{\dagger}$ & GBM & Yes & PWI & GBM \\
\hline 6 & GBM & GBM & No & PWI & GBM \\
\hline 13 & $\mathrm{GBM}^{*} /$ metastasis & GBM & Yes & PWI, MRS & GBM \\
\hline 17 & Metastasis $^{\dagger}$ & Multifocal glioma & Yes & MRS & Multifocal GBM \\
\hline 19 & GBM/lymphoma* & GBM & Yes & PWI, MRS & GBM \\
\hline 20 & GBM/metastasis* & GBM & Yes & PWI, MRS & GBM \\
\hline 24 & Metastasis/GBM ${ }^{*}$ & Metastasis $^{\dagger}$ & No & MRS & GBM \\
\hline 28 & Meningioma/GBM ${ }^{*}$ & GBM & Yes & PWI, MRS & GBM \\
\hline 34 & GBM & GBM & No & PWI, MRS & GBM \\
\hline 35 & Lymphoma ${ }^{\star} / G B M$ & GBM & Yes & PWI, MRS & GBM \\
\hline 37 & GBM & GBM & No & PWI, MRS & GBM \\
\hline 41 & GBM & GBM & No & PWI, MRS & GBM \\
\hline 33 & Astrocytoma, low-grade & Astrocytoma, low-grade & No & PWI, MRS & Astrocytoma grades I - II \\
\hline 12 & Astrocytoma, low-grade & Astrocytoma, high grade ${ }^{\dagger}$ & No & PWI, MRS & Astrocytoma grade II \\
\hline 22 & Astrocytoma, low-grade & Astrocytoma, low-grade & No & PWI, MRS & Astrocytoma, grade II \\
\hline 23 & Astrocytoma, low-grade, ${ }^{*}$ encephalitis & Astrocytoma, low-grade & Yes & MRS & Astrocytoma, grade II \\
\hline 25 & Astrocytoma, high-grade & Astrocytoma, high grade & No & PWI, MRS & $\begin{array}{l}\text { Astrocytoma grades } \\
\text { II - III }\end{array}$ \\
\hline 27 & $\begin{array}{l}\text { Low-grade astrocytoma/ } \\
\text { oligodendroglioma }\end{array}$ & Astrocytoma, high-grade & No & PWI, MRS & Astrocytoma grade II - III \\
\hline 2 & Astrocytoma, low-grade ${ }^{\dagger}$ & Astrocytoma, high-grade & Yes & PWI & Astrocytoma grade III \\
\hline 7 & Astrocytoma, high-grade & Astrocytoma, high-grade & No & PWI & Astrocytoma grade III \\
\hline 8 & Astrocytoma, low-grade ${ }^{\dagger}$ & Astrocytoma, high-grade & Yes & PWI, MRS & Astrocytoma grade III \\
\hline 1 & Metastasis/GBM ${ }^{*}$ & Metastasis & Yes & PWI & Metastasis \\
\hline 9 & Metastasis & Metastasis & No & PWI & Metastasis \\
\hline 10 & Metastasis & Abscess $^{\dagger}$ & No & PWI & Metastasis \\
\hline 14 & Metastasis & Metastasis & No & MRS & Metastasis \\
\hline 15 & Infarct $/$ metastasis* & Metastasis & Yes & MRS & Metastasis \\
\hline 16 & GBM with bleeding ${ }^{\dagger}$ & Metastasis & Yes & MRS & Metastasis \\
\hline 36 & Meningioma/metastasis ${ }^{*}$ & Metastasis & Yes & PWI, MRS & Metastasis \\
\hline 39 & GBM/metastasis* & Metastasis & Yes & PWI, MRS & Metastasis \\
\hline 43 & GBM/metastasis* & Metastasis & Yes & PWI, MRS & Metastasis \\
\hline 3 & GBM/metastasis* & Lymphoma & Yes & PWI & Lymphoma \\
\hline 18 & Lymphoma/GBM ${ }^{*}$ & Lymphoma & Yes & PWI, MRS & Lymphoma \\
\hline 21 & GBM/lymphoma* & Lymphoma & Yes & PWI, MRS & Lymphoma \\
\hline 11 & Meningioma & Meningioma & No & PWI & Meningioma \\
\hline 42 & Meningioma ${ }^{\star} /$ meningeal metastasis/ & Meningioma & Yes & PWI, MRS & Meningioma \\
\hline 26 & DNET & DNET & No & MRS & DNET \\
\hline 30 & $\begin{array}{l}\text { Ganglioglioma/ high-grade } \\
\text { astrocytoma }^{*}\end{array}$ & $\begin{array}{l}\text { Low-grade glial tumour, } \\
\text { ganglioglioma }\end{array}$ & Yes & PWI, MRS & Ganglioglioma \\
\hline 32 & Metastasis/malignant glioma* & Malignant glial tumour & Yes & PWI, MRS & Oligodendroglioma III \\
\hline 40 & 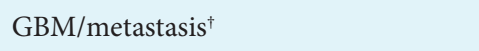 & Oligodendroglioma & Yes & PWI, MRS & Oligodendroglioma III \\
\hline 44 & Oligodendroglioma & Oligodendroglioma & No & PWI, MRS & Oligodendroglioma \\
\hline 29 & Astrocytoma/low-grade ${ }^{\dagger}$ & Ischaemia/vasculitis & Yes & MRS & Vasculitis \\
\hline 31 & High-grade astrocytoma $^{\dagger}$ & Radiation injury & Yes & MRS & Radiation injury \\
\hline 38 & GBM/metastasis/abscess ${ }^{\star}$ & Abscess & Yes & MRS & Abscess \\
\hline
\end{tabular}


The use of multimodal MRI enabled the concept of establishing criteria for the grading of gliomas, ${ }^{[3,6,8,9]}$ the differentiation of metastases from glial tumours, ${ }^{[10,11]}$ and the differentiation of lymphomas from other brain tumours. ${ }^{[12-14]}$ To the best of our knowledge, there are limited data on the added utility of multimodal MRI (performed at 3 Tesla MR field strength) for accurate diagnosis, compared with that obtained by biopsy, or comparing the diagnostic accuracy of conventional MRI with that of multimodal MRI, when the aforementioned proposed criteria are used in the evaluation.

Most reports in the literature on the role of MRS and PWI in brain tumours present the differences in the mean values for the rCBV and the metabolite ratio on MRS in different types of tumour. The aim of this study was not to present new cut-off values for rCBV or metabolite ratios in different types of brain tumour, but rather to determine whether multimodal MRI, using some of the criteria and cut-off values published in the literature, can increase the diagnostic accuracy in brain tumours over MRI performed with only conventional sequences.

\section{Materials and methods}

The study included a retrospective analysis of MRIs of 44 consecutive patients with suspected brain tumours from 2 institutions, who were examined with conventional MR sequences including DWI and with PWI and/or MRS. All examinations were performed on a 3 Tesla MR scanner (TRIO, Siemens AG, Medical Solutions, Erlangen, Germany) using an 8-channel head coil. Twenty-five patients underwent both PWI and MRS, 9 patients underwent only PWI, and 10 patients underwent only MRS (Table 1). All patients underwent surgical removal of their tumour, surgical debulking, or surgical removal of a portion of tumour to obtain a histopathological diagnosis. The histopathological diagnosis was considered the 'gold standard' in this study.

\section{Conventional MRI}

The MR protocol included the following sequences: a 3-plane localiser; sagittal T1WI (T1-weighted images; TR/TE: 400/10); axial T1WI (TR/ TE: 550/10); axial FLAIR images (fluid-attenuated inversion-recovery images; TR/TE: 9900/125); and axial T2WI (TR/TE: 3700/90). Following contrast administration, axial and coronal T1WI views were also obtained. All sequences were obtained with a $220 \mathrm{~mm}$ field of view and an interpolated $256 \times 256$ matrix. Slice thicknesses were $5 \mathrm{~mm}$ for axial images and $4 \mathrm{~mm}$ for sagittal and coronal images with a 1 $\mathrm{mm}$ intersection gap. DWI was obtained in all patients. The DWI was performed with the diffusion gradients applied along each of the 3 principal directions $(x, y, z)$ with a diffusion sensitivity (b-values) of 0.500 and $1000 \mathrm{~s} / \mathrm{mm}^{2}$, respectively. Three sets of axial diffusionweighted images were generated, and an apparent diffusion coefficient (ADC) map was calculated.

\section{MR perfusion (PWI)}

PWI (T2*-weighted, gradient-echo single-shot EPI sequence) was performed during a single bolus contrast infusion. A gadoliniumbased MR contrast agent, at a dose of $0.1 \mathrm{mmol}$ per kg of body weight, was infused with a power injector at a rate of $5 \mathrm{ml} / \mathrm{second}$, followed by a $40 \mathrm{ml}$ normal saline flush at the same infusion rate. Scan parameters were as follows: TR/TE 2 410/49 ms; flip angle 90 degrees; field of view $220 \times 220 \mathrm{~mm}^{2}$; and acquisition time 120 seconds. Twentytwo slices were obtained with slice thickness of $5 \mathrm{~mm}$, with $1.5 \mathrm{~mm}$ intersection gap, and the matrix was $128 \times 128$. Perfusion analysis, based on deconvolution using singular value decomposition, ${ }^{[15,16]}$ was performed at a Leonardo workstation using the 'Perfusion MR' application (Siemens AG, Medical Solutions, Erlangen, Germany). Relative regional cerebral blood volume (rCBV) maps were generated and included in the analysis. The rCBV values in the tumour and in the normal-appearing white matter (NAWM) in the centrum semiovale were determined. The $\mathrm{rCBV}_{\text {tumour }}$ was the maximum $\mathrm{rCBV}$ value obtained in the tumour. The peritumoural rCBV was measured in the peritumoural oedema, which was defined as the brain tissue with high signal intensity on FLAIR and with no contrast enhancement on $\mathrm{T} 1 \mathrm{~W}$ after contrast administration. The $\mathrm{rCBV}_{\text {ratio }}$ was calculated as the $\mathrm{rCBV}_{\text {tumour }} / \mathrm{rCBV}_{\mathrm{NAWM}}$. However, concentration-time curves were not calculated as dynamic susceptibility contrast was not available in our MR system.

\section{MR spectroscopy (MRS)}

An automated, multivoxel 2D chemical shift imaging (CSI) was obtained (TR/TE: 1 500/135, flip angle 90, bandwidth 1200 , vector size 1024 , acquisition time 6 minutes and 53 seconds, and a total of 3 averages). The CSI sequence produced an interpolated $16 \times 16$ transversely orientated matrix, defined by phase encoding, with a FOV of $16 \mathrm{~cm}$. The field homogeneity was achieved by automated multiple-angle projection (MAP) shimming. Spectral maps were collected with $2.56 \mathrm{~ms}$ RF pulses for chemical shift-selective excitation (CHESS) and subsequent spoiling of the resultant water signal. All spectra were acquired after contrast administration in order to obtain clear delineation of the tumour margins. The volume of VOIs of $8 \times 8 \times 1.5 \mathrm{~cm}^{3}$ (a total of 64 VOIs; each $1 \mathrm{~cm}$ large) was placed individually in every patient, depending on tumour volume and location. The volume of VOIs was carefully placed to include the tumour, the surrounding oedema, normal brain tissue on the same side of the tumour, and the normal brain tissue in the contralateral hemisphere. In large tumours, two such VOIs-volume were applied. Normal brain tissue was defined as brain tissue with no signal changes on FLAIR and with no contrast enhancement on T1W after contrast administration. All examinations were analysed using the 'Spectroscopy' application on a Leonardo workstation (Siemens AG, Medical Solutions, Erlangen, Germany). This application calculated the peak areas for the metabolites, enabled calculation of the ratio between different metabolites, and provided a colour map of the whole ROI, as well as colour maps of single metabolites and the ratio between two different metabolites. The metabolite ratios considered during the evaluation were the most pathological values in all the VOIs studied for every individual patient.

\section{Evaluation of the images}

Evaluation of all images was performed, in consensus, by two experienced neuroradiologists. A 2-step evaluation of the images from conventional MR sequences including DWI was performed: (i) first, the readers were allowed to suggest 2 - 3 differential diagnoses per examination; and then ( $i i)$ the readers were allowed to suggest only 1 possible diagnosis per MR examination. In a third, final evaluation, all images, including PWI and MRS, were assessed. At this assessment session, only 1 diagnosis could be suggested for each individual examination. 
Table 2. Compatibility of the diagnosis provided by conventional MRI sequences and multimodal MRI with the diagnosis obtained at biopsy

\begin{tabular}{|c|c|c|c|}
\hline & $\begin{array}{l}\text { Yes } \\
n(\%)\end{array}$ & $\begin{array}{l}\text { No } \\
n(\%)\end{array}$ & $p$-value \\
\hline Single given diagnosis was the same as the final histopathological diagnosis obtained at biopsy & & & $<0.001$ \\
\hline Conventional MRI sequences & $17(39)$ & $27(61)$ & \\
\hline +PWI, MRS, DWI & $41(93)$ & $3(7)$ & \\
\hline The final histopathological diagnosis was among 2 - 3 suggested differential diagnoses & & & 0.118 \\
\hline Conventional MRI sequences & $35(79)$ & $9(21)$ & \\
\hline +PWI, MRS, DWI & $41(93)$ & $3(7)$ & \\
\hline
\end{tabular}

\section{Criteria for grading gliomas and differentiating gliomas from other tumours}

The following radiological criteria were used in the assessment of multimodal MRI:

(a) tumours with contrast enhancement, an $\mathrm{rCBV}_{\text {ratio }}>1.5$ on PWI, a choline (Cho)/creatine $(\mathrm{Cr})>2.5$ in the tumour on MRS, and an $\mathrm{N}$-acetylaspartate (NAA)/cho $<1$ in the normal-appearing brain tissue adjacent to the tumours were considered to be highly malignant gliomas $^{[3,10,17]}$

(b) tumours with no contrast enhancement, an $\mathrm{rCBV}$ ratio $<1.5$ on $\mathrm{PWI},{ }^{[18]}$ and with a cho/cr $<2.5$ on MRS were considered to be lowmalignant gliomas ${ }^{[3,17]}$

(c) tumours with homogeneous contrast enhancement and with an $\mathrm{rCBV}_{\text {ratio }}<1.5$ on PWI were considered to be CNS lymphomas ${ }^{[12,14]}$

(d) tumours with contrast enhancement (homogeneous or ringenhancing tumours), an $\mathrm{rCBV}_{\text {ratio }}>1.5$ on $\mathrm{PWI}$, a cho/cr $>2.5$ in the tumour on MRS, and an NAA/cho $>1$ in the normalappearing brain tissue adjacent to the tumour were considered to be metastases ${ }^{[10,12,14]}$

(e) peritumoural rCBV: Tumours with low $\mathrm{rCBV}$ in the immediate peritumoural tissue (with no contrast enhancement) were considered to be metastases, while those with high $\mathrm{rCBV}$ were considered to be highly malignant gliomas ${ }^{[19,20]}$

(f) cystic lesions with rim contrast enhancement, low ADC value in the central cystic portion, and with lactate and amino acid peaks on MRS, were considered to be abscesses.

Criteria (d) and (e) were considered together when differentiating between metastases and highly malignant gliomas in patients who underwent both PWI and MRS. As both tumours showed high intratumoural $\mathrm{rCBV}$ and cho/cr, metastases exhibited low peritumoural rCBV, normal or near-normal cho/cr and NAA/Cho $>1$ in the normalappearing brain tissue adjacent to the tumour.

\section{Statistical analysis}

Statistical analysis was performed with SPSS 17 to compare the compatibility of the diagnosis provided by multimodal MRI and that provided by conventional MR sequences, with the final histopathological diagnosis obtained by biopsy. The concordance between the histopathological diagnosis and the diagnosis provided by conventional MRI and multimodality MRI was estimated by calculating the kappa coefficient (Cohen's kappa). Interpretation of the kappa coefficient ( $\kappa$
Table 3. The added utility of multimodal MRI

\begin{tabular}{lll}
\hline $\begin{array}{l}\text { Multimodality MRI helped } \\
\text { differentiation between: }\end{array}$ & $\boldsymbol{n}$ & Modality \\
\hline Lymphoma and GBM & 7 & PWI (6) \\
GBM and metastases & 7 & MRS (6) \\
& & PWI (1) \\
High-grade and low-grade glial & 5 & PWI (1) \\
tumour & & MRS (4) \\
GBM and other tumours & 2 & MRS \\
Metastasis and meningioma & 2 & MRS \\
Tumour and infarction & 1 & MRS \\
Tumour and encephalitis & 1 & MRS \\
Tumour and radiation injury & 1 & MRS \\
Tumour and abscess & 1 & MRS and \\
Total, $N$ & & DWI
\end{tabular}

values) was performed according to the method proposed by Landis. ${ }^{[21]}$ $\mathrm{A} \kappa$ of 1 indicates total agreement, whereas a $\kappa$ of 0 means poor agreement and indicates that any observed agreement is attributable to chance. A $\kappa$ of $0.81-1.00$ indicates almost perfect agreement, $0.61-0.80$ indicates substantial agreement, $0.41-0.60$ indicates moderate agreement, 0.21 0.40 indicates fair agreement, $0-0.20$ indicates slight agreement, and $<0$ indicates poor agreement. To estimate the added utility of multimodal MRI, Fisher's exact test and/or chi-square test was performed. Differences with a $p$-value $<0.05$ were considered statistically significant.

\section{Results}

\section{Patients}

This retrospective study included 44 patients with suspected brain tumours, 27 (61\%) male and 17 (39\%) female. Their mean age was 58 $(\mathrm{SD} \pm 17$ ) years, and median and range were 63.5 and 23 - 87 years, respectively. The final diagnoses obtained by biopsy are shown in Table 1. Glioblastoma multiforme ( $n=13,29.5 \%$ of the study population) was the most commonly encountered diagnosis, followed by metastases $(n=9$, $20.5 \%)$, and astrocytomas grade I - III ( $n=9,20.5 \%)$. CNS lymphoma was the final diagnosis in 3 patients. Other tumours were the final diagnosis in 7 patients ( 3 oligodendrogliomas, 2 meningiomas, 1 dysembryoplastic 


\section{ORIGINAL ARTICLE}

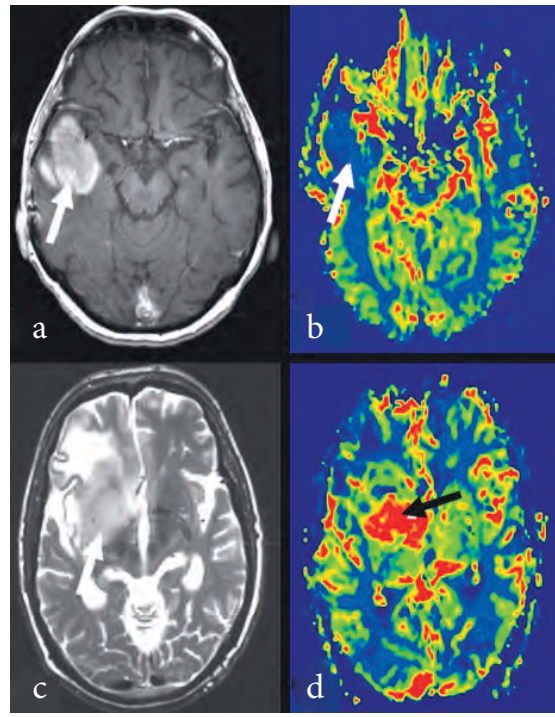

Fig. 1. $(a-b)$ A 71-year-old man with CNS lymphoma. (a) Axial post-contrast T1WI, and (b) CBV map of perfusion-weighted images showing homogeneous contrast enhancement (arrow, a), and low CBV (arrow, b). (c-d) A 65-year-old man with glioblastoma multiforme. (c) T2WI, and (d) CBV map of perfusion-weighted images showing high signal intensity on T2WI and high CBV on PWI (arrow).

neuroepithelial tumour (DNET) and 1 ganglioglioma) while 'tumour-like lesions' was the final diagnosis in 3 lesions initially believed to be brain tumours on conventional MRI (infarction secondary to vasculitis, radiation injury and brain abscess).

\section{Concordance between multimodal MRI diagnosis and final diagnosis}

The concordance between the diagnosis provided by evaluating images from multimodal MRI and the final diagnosis was almost perfect $(\kappa$ value $0.92 ; 95 \% \mathrm{CI}$ $0.82-1)$, whereas the concordance between the diagnosis provided by the evaluation of images from conventional MRI and the final diagnosis was only fair ( $\kappa$ value 0.38 ; $95 \%$ confidence interval $0.20-0.56$ ).

\section{Added utility of multimodal MRI} The single suggested diagnosis obtained with multimodal MRI was compatible with the final diagnosis obtained at biopsy in 41 (93\%) patients, compared with a lower rate in $17(39 \%)$ patients when the readers were allowed to give only 1 diagnostic possibility during the evaluation of the routine MR sequences. This difference was statistically

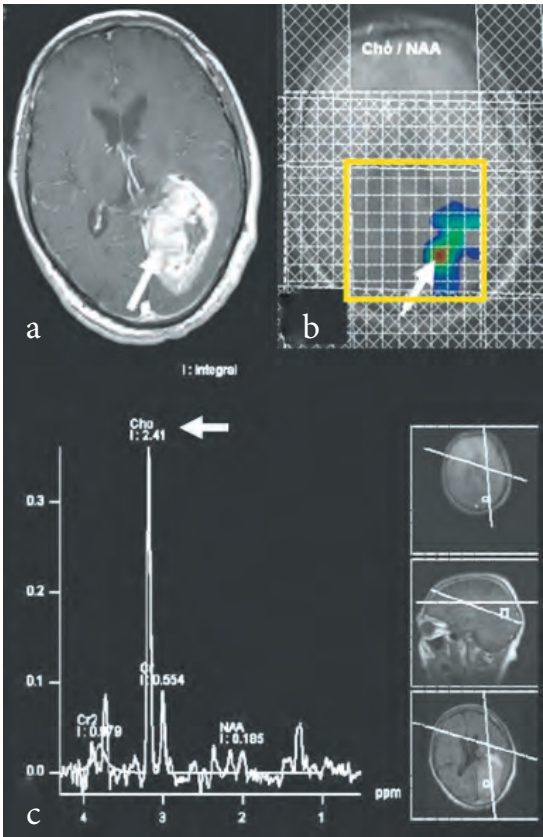

Fig. 2. A 67-year-old man with GBM. (a) Axial postcontrast T1WI. (b) MR spectroscopy colour map. (c) MR spectrum showing a tumour with strong enhancement (arrow, a) with central necrosis. The colour map shows the maximum choline/creatine ratio in the inner portion of the tumour (arrow, $b$ ). Metabolite spectrum shows a very high choline peak (arrow, c) with a choline/NAA ratio of 23 and a choline/creatine ratio of 4.3, typical of a malignant glial tumour. These ratios were also high in the oedema anterior to the tumour, which helped to differentiate GBM from solitary metastasis. The yellow square in (b) shows the volume of VOIs of 8 $x 8 \times 1.5 \mathrm{~cm}^{3}$ (total: $64 \mathrm{VOIs}$, each $1 \mathrm{~cm}$ large) used in $2 \mathrm{D}$ chemical shift imaging.

significant $(p<0.001)$ (Table 2$)$. When the readers were allowed to give more than 1 diagnostic possibility (maximum of 3 differential diagnoses), the compatibility of the diagnoses provided by the routine MR sequences with the final histopathological diagnosis obtained at biopsy increased to $79 \%$ ( $p=0.118)$ (Table 2).

Compared with the differential diagnoses suggested when evaluating the conventional MRI, the multimodal MRI (MRS and/or PWI) provided additional information in 27 (61\%) patients and helped to differentiate between lymphoma and GBM in 7 patients (4 GBM and 3 lymphomas); between GBM and metastases in 7 patients; between high-grade and low-grade astrocytomas in 5 patients; between various other tumours in 4 patients; and between tumours and non-neoplastic conditions in 4 patients (Table 3 ).

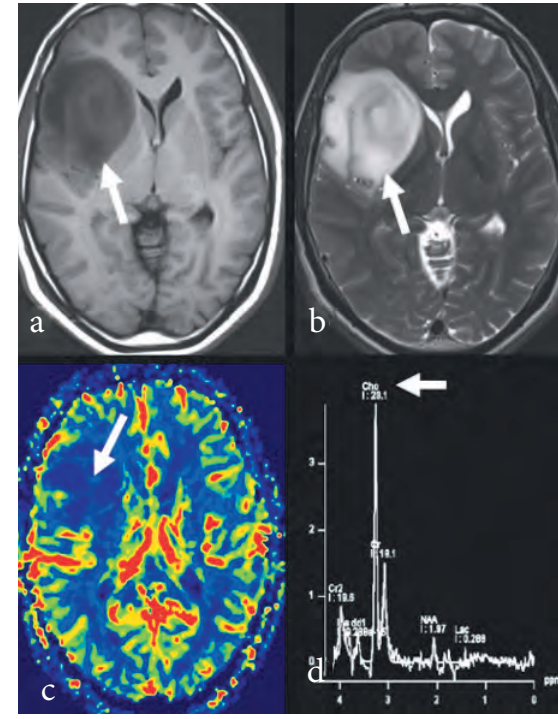

Fig. 3. A 30-year-old man with low-grade astrocytoma. ( $a-d)$ Axial T1WI, axial T2WI, CBV map and MR spectroscopy of a patient with a large tumour in the right frontal lobe (arrows $a$, b). PWI shows low CBV (arrow, c) and MR of 1.5. Despite a very high choline/NAA ratio of 14.3 , the radiological diagnosis was low-grade astrocytoma, which was also the histopathological diagnosis obtained at biopsy.

PWI was most useful in differentiating between CNS lymphomas and other tumours in 7 patients (between CNS lymphoma and GBM in 6 patients (Fig. 1) and between low-grade and high-grade astrocytoma in 1 patient) (Table 3). PWI was helpful in differentiating 1 metastasis from GBM in 1 patient (patient no. 1) in whom the peritumoural rCBV was very low. MRS was useful in differentiating between GBM and metastases (Fig. 2) in 6 patients as well as between low-grade and high-grade astrocytoma (Fig. 3) in 4 patients (Table 3).

Among patients who only underwent PWI $(n=9)$, this modality had an added utility in only 4 (44\%) patients, whereas in patients who only underwent MRS $(n=10)$, this modality had an added utility in 7 (70 \%) (Table 1)

\section{Discussion}

In patients with brain lesions, an accurate diagnosis is fundamental for an accurate therapy choice, to avoid unnecessary brain surgery, and to prevent delay in initiating treatment. Studies on diagnostic accuracy have shown that MRI is superior to contrastenhanced CT in the diagnosis of brain metastases. ${ }^{[22]}$ Combining MRI and MRS 
has been shown to provide better diagnostic value than MRS alone, especially in contrast-enhancing tumours. ${ }^{[23]}$ Another study on the diagnostic accuracy, sensitivity and specificity of diagnostic imaging strategies to differentiate various intra-axial brain masses also showed a high accuracy for multimodal imaging. ${ }^{[24]}$ In contrast to our study, the histological findings, laboratory findings or clinical diagnosis were the reference standard in the aforementioned retrospective study, the field strength for MRI was 1.5 Tesla, and the MRS was performed using single voxel technique. ${ }^{[24]}$ To our knowledge, there are limited data for diagnostic accuracy and the added utility using a multimodal MRI performed on 3 Tesla MR field strength and using MRS with CSItechnique in the differentiation of different types of brain tumour.

Law et $a l .{ }^{[17]}$ showed that a combination of the estimation of $\mathrm{rCBV}, \mathrm{Cho} / \mathrm{Cr}$ and Cho/NAA in 160 gliomas resulted in a sensitivity, specificity, PPV and NPV of $93.3 \%, 60.0 \%, 87.5 \%$ and $75.0 \%$ respectively, in grading these tumours, compared with a sensitivity, specificity, PPV and NPV of $72.5 \%, 65.0 \%, 86.1 \%$ and $44.1 \%$ respectively, in grading when only conventional MRI images were evaluated. Our study showed that the diagnostic accuracy of MRI increases when a multimodal MRI approach is used in the work-up of brain tumours. In some tumours, e.g. lymphomas and some types of metastases, this finding might help in planning tumour therapy, without the need for biopsy. Solid tumours, such as lymphomas with high cellularity as the predominant feature, usually exhibit low $\mathrm{rCBV}$, and PWI can help to differentiate these tumours from solid malignant tumours, such as glioma and metastases. ${ }^{[14]}$

As in previous reports, ${ }^{[12-14]}$ PWI in our study played an important role in differentiating CNS lymphoma (low rCBV) from GBM and metastases (high rCBV). Even meningiomas exhibit high rCBV and often show homogeneous contrast enhancement. However, the diagnosis of 1 case of meningioma in our study was confirmed by conventional MR sequences, depending on the presence of a dural tail and the relation of the tumour to the adjacent falx (patient no. 11). As shown in other studies, a high-grade brain tumour shows greater rCBV than a low-grade tumour. ${ }^{[12,25]}$ In our study, PWI helped to suggest a high-grade glioma in a patient (patient no. 2) upon follow-up of her low-grade glioma, by showing a high $\mathrm{rCBV}$ in tumour areas despite the absence of contrast enhancement. Law et al. ${ }^{[19]}$ and Chiang et al. ${ }^{[20]}$ showed low peritumoural $\mathrm{rCBV}$ in metastases compared with highly malignant gliomas. However, the cut-off values for rCBV in gliomas v. metastases suggested by Chiang et al. were almost twice as high as those suggested by Law et al., with considerable overlap between the values for gliomas and metastases. Based on these previous results, we performed our own analysis of the rCBV values in the peritumoural oedema and calculated the ratio between $\mathrm{rCBV}$ in peritumoural oedema and $\mathrm{rCBV}$ in the NAWM, as measured in the centrum semiovale. We found that the ratio of $\mathrm{rCBV}$ in peritumoural oedema to $\mathrm{rCBV}$ in NAWM was $1 \pm 0.5$ in GBM, compared with $0.45 \pm 0.3$ in metastases. We believe that the determination of the $\mathrm{rCBV}_{\text {ratio }}$ for the peritumoural $\mathrm{rCBV}$, and possibly even the intratumoural rCBV, may help to avoid the aforementioned differences in the reference values. As the number of patients included in our study was small, this criterion needs to be validated in larger studies.

Similar to previous reports, our study showed that MRS can help to differentiate between primary brain tumours and metastases by demonstrating high cho/cr and cho/NAA in the tissue surrounding the area of contrast enhancement in primary brain tumours, compared with a normal ratio in the vasogenic oedema surrounding metastatic lesions. ${ }^{[10]}$ as well as demonstrating the occurrence of a lipid peak in metastases. ${ }^{[11]}$ In common with other studies, ${ }^{[8,9]}$ MRS helped to suggest the diagnosis of a high-grade astrocytoma by showing a cho/cr ratio exceeding 2.5 in 2 tumours previously believed to be low-grade tumours (patients no. 5 and 8; Table 1). However, 1 false-positive and false-negative result occurred in our study. In one case, a high-grade astrocytoma was suggested by MRS in one patient with a known low-grade astrocytoma (patient no. 12), but a new biopsy showed no evidence that the tumour should be upgraded, based on the obtained biopsy material. One patient considered to have metastasis on MRS (patient no. 24) was shown to have GBM at biopsy, while another patient (patient no. 10) showed low ADC values on DWI and was considered to have a brain abscess. This patient underwent only PWI, and biopsy showed metastasis from a mucinous adenocarcinoma.

Although the diagnosis obtained by biopsy is considered the gold standard, this procedure provides information about only a limited area of neoplastic tissue and does not provide any histological data on the remaining tumour tissue not included in the biopsy material. This is especially important in grading gliomas, where we believe that multimodal MRI provides more comprehensive information about the whole tumour. MR spectroscopy can play a useful role after treatment in assessing the therapeutic response, as choline decreases and lactate and/or lipids may increase after therapy. ${ }^{[6,27]}$ This is particularly important in the detection of treatment failure, and may enable modifying the treatment strategy to control significant progression of the disease.

The reason why 3 patients with final diagnosis of vasculitis, radiation injury and brain abscess (patients no. 29, 31 and 38, respectively) were included in the study was that the suspicion of tumour was very high, based on conventional MRI, and could therefore not be excluded before obtaining biopsy, despite the spectroscopic suggestion of a nonneoplastic condition. This confirms the added utility of multimodal MRI in differentiating tumours from tumour like-lesions.

One of the limitations of the study was its retrospective nature, which meant that only 25 patients underwent both PWI and MRS. We believe that the added utility of multimodal MRI and the concordance between the diagnosis provided by evaluating images from multimodal MRI and the final diagnosis might have been further increased if all patients had been examined with both modalities. Our findings of lower added utility of MRS (70\%) and PWI (44\%) when only 1 of the modalities was performed, indicate that the findings of these modalities often are supportive of each other. To provide better delineation of the tumour, MRS was performed after the introduction of contrast agent, which is another limitation of this study as contrast agent might have contributed to some modulation of the choline signal. ${ }^{[28]}$ Finally, permeability issues and extravasation of contrast agent could also have affected perfusion levels in contrast-enhancing tumours, as dynamic susceptibility contrast sequence was not obtained.

The disadvantages of multimodal MRI include the increased cost of the MR examination, increased examination time, increased postprocessing and reading time, and the need for an experienced neuroradiologist who is familiar with the postprocessing and interpretation of images and metabolic spectra produced by these modalities. 


\section{Conclusion}

Combining PWI and MRS with conventional MR imaging increases the diagnostic accuracy of MRI in the evaluation of brain tumours and tumour-like lesions. In some tumours, this benefit might preclude brain biopsy, which is an invasive procedure that would otherwise be required to establish the final diagnosis; it might also avoid delay in initiating tumour therapy. Although expensive and time-consuming, multimodality MRI examinations should, wherever available, be performed in the work-up of brain tumours and tumour-like lesions.

Author contributions. KK contributed to the conception and design of the study, acquisition of data, analysis and interpretation of data and drafting the manuscript, and has given his final approval of the version to be published. MT has contributed in acquisition of data, revising the manuscript critically for important intellectual content, and has given her final approval of the version to be published. SP has contributed to revision of the manuscript critically for important intellectual content, and has given his final approval of the version to be published. PS has contributed to the conception and design, analysis and interpretation of data, and revising the manuscript critically for important intellectual content, and has given her final approval of the version to be published.

1. Adamson C, Kanu OO, Mehta AI, et al. Glioblastoma multiforme: A review of where we have been and where we are going. Expert Opin Investig Drugs 2009;18(8):1061-1083. [http://dx.doi. org/10.1517/13543780903052764]

2. van den Bent MJ, Afra D, de Witte $\mathrm{O}$, et al. Long-term efficacy of early versus delayed radiotherapy for low-grade astrocytoma and oligodendroglioma in adults: the EORTC 22845 randomised trial. Lancet 2005;366(9490):985-990

3. Zonari P, Baraldi P, Crisi G. Multimodal MRI in the characterization of glial neoplasms: The combined role of single-voxel MR spectroscopy, diffusion imaging and echo-planar perfusion imaging. Neuroradiology 2007;49(10):795-803.

4. Fayed N, Davila J, Medrano J, et al. Malignancy assessment of brain tumours with magnetic resonance spectroscopy and dynamic susceptibility contrast MRI. Eur J Radiol 2008;67(3):427-433. [http://dx.doi. org/10.1016/j.ejrad.2008.02.039]

5. Pomper MG, Port JD. New techniques in MR imaging of brain tumours. Magn Reson Imaging Clin N Am 2000;8(4):691-713.

6. Spampinato MV, Smith JK, Kwock L, et al. Cerebral blood volume measurements and proton MR spectroscopy in grading of oligodendroglial tumours. Am J Roentgenol 2007;188(1):204-212.

7. Poptani H, Gupta RK, Roy R, et al. Characterization of intracranial mass lesions with in vivo proton MR spectroscopy. Am J Neuroradiol 1995;16(8):1593-1603.

8. Preul MC, Caramanos Z, Leblanc R, et al. Using pattern analysis of in vivo proton MRSI data to improve the diagnosis and surgical management of patients with brain tumours. NMR Biomed 1998;11(45):192-200.
9. Dowling C, Bollen AW, Noworolski SM, et al. Preoperative proton MR spectroscopic imaging of brain tumours: Correlation with histopathologic analysis of resection specimens. Am J Neuroradiol 2001;22(4):604-612.

10. Burtscher IM, Holtas S. Proton magnetic resonance spectroscopy in brain tumours: Clinical applications. Neuroradiology 2001;43(5):345-352

11. Moller-Hartmann W, Herminghaus S, Krings T, et al. Clinical application of proton magnetic resonance spectroscopy in the diagnosis of intracranial mass lesions. Neuroradiology 2002;44(5):371-381.

12. Hakyemez B, Erdogan C, Bolca N, et al. Evaluation of different cerebral mass lesions by perfusionweighted MR imaging. J Magn Reson Imaging 2006;24(4):817-824

13. Sugahara T, Korogi Y, Shigematsu Y, et al. Perfusion-sensitive MRI of cerebral lymphomas: a preliminary report. J Comput Assist Tomogr 1999;23(2):232-237.

14. Abul-Kasim K, Maly P, Strömbeck A, et al. Perfusion weighted MR imaging may differentiate primary CNS-lymphoma from other homogeneously enhancing brain tumours. The Neuroradiology Journal 2008;21:637-644.

15. Ostergaard L, Weisskoff RM, Chesler DA, et al. High resolution measurement of cerebral blood flow using intravascular tracer bolus passages. Part I: Mathematical approach and statistical analysis. Magn Reson Med 1996;36(5):715-725.

16. Ostergaard L, Sorensen AG, Kwong KK, et al. High resolution measurement of cerebral blood flow using intravascular tracer bolus passages. Part II: Experimental comparison and preliminary results. Magn Reson Med 1996;36(5):726-736.

17. Law M, Yang S, Wang H, et al. Glioma grading: sensitivity, specificity, and predictive values of perfusion MR imaging and proton MR spectroscopic imaging compared with conventional MR imaging. Am J Neuroradiol 2003;24(10):1989-1998.

18. Yang D, Korogi $Y$, Sugahara $T$, et al. Cerebral gliomas: prospective comparison of multivoxel 2D chemical-shift imaging proton MR spectroscopy, echoplanar perfusion and diffusion-weighted MRI. Neuroradiology 2002;44(8):656-666.

19. Law M, Cha S, Knopp EA, et al. High-grade gliomas and solitary metastases: differentiation by using perfusion and proton spectroscopic MR imaging. Radiology 2002;222(3):715-721.

20. Chiang IC, Kuo YT, Lu CY, et al. Distinction between high-grade gliomas and solitary metastases using peritumoural 3-T magnetic resonance spectroscopy, diffusion, and perfusion imagings. Neuroradiology 2004;46(8):619-627.

21. Landis JR, Koch GG. The measurement of observer agreement for categorical data. Biometrics 1977;33(1):159-174.

22. Schellinger PD, Meinck HM, Thron A. Diagnostic accuracy of MRI compared to CCT in patients with brain metastases. J Neurooncol 1999;44(3):275-281

23. Galanaud D, Nicoli F, Chinot O, et al. Noninvasive diagnostic assessment of brain tumours using combined in vivo MR imaging and spectroscopy. Magn Reson Med 2006;55(6):1236-1245

24. Al-Okaili RN, Krejza J, Woo JH, et al. Intraaxial brain masses: MR imaging-based diagnostic strategy-initial experience. Radiology 2007;243(2):539-550.

25. Roberts HC, Roberts TP, Brasch RC, et al. Quantitative measurement of microvascular permeability in human brain tumours achieved using dynamic contrast-enhanced MR imaging: Correlation with histologic grade. Am J Neuroradiol 2000;21(5):891-899.

26. Shino A, Nakasu S, Matsuda M, et al. Noninvasive evaluation of the malignant potential of intracranial meningiomas performed using proton magnetic resonance spectroscopy. J Neurosurg 1999;91(6):928934

27. Graves EE, Nelson SJ, Vigneron DB, et al. Serial proton MR spectroscopic imaging of recurrent malignant gliomas after gamma knife radiosurgery. Am I Neuroradiol 2001;22(4):613-624.

28. Madhu B, Robinson SP, Howe FA, et al. Effect of Gd-DTPA-BMA on choline signals of HT29 tumours detected by in vivo 1H MRS. J Magn Reson Imaging 2008;28(5):1201-1208. [http://dx.doi.org/10.1002/ jmri.21577 